\title{
Research of Integrated Test System of Potentiometer Electric Parameter Based on Multi-CPU Control
}

\author{
Yuan Jingping ${ }^{1, a^{*}}$, Zhaoxiaorong ${ }^{1, b}$, Yu Fenfen ${ }^{1, c}$ \\ ${ }^{1}$ Jiangsu University of Technology, China \\ ayuan_jp@jsut.edu.cn, bzhaoxr@jsut.edu.cn, yff@jsut.edu.cn
}

Keywords: Potentiometer, Integrated Test System, Multi-CPU, STC Microcontroller

\begin{abstract}
The problems of low testing efficiency and high testing cost exist in small and medium potentiometer enterprises when multi-station and multi-instrument are used to respectively test potentiometer electric parameters. The paper propose a designing project based on the integrated test system of multi-CPU, analyzes the working principle of the system and introduces in detail the software and hardware design. The result of the experiment indicates that this system not only enjoys high test precision and fast speed, but also reduces effectively the testing cost.
\end{abstract}

\section{Introduction}

Potentiometer is a commonly used electronic component so it is widely applied in the circuit for its remarkable adjustability. The important electric parameters of a potentiometer include: nominal resistance (total resistance), the allowable deviation of nominal resistance, zero resistance, dynamic noise, etc., and for duplex potentiometer, synchronization error is one of the important electric parameters. At present, most small and medium-sized production lines use multi-station and multi-instrument to test a variety of electric parameters of the potentiometer respectively, which results in high cost and low efficiency. Thus we suggest using one instrument on one workstation to efficiently complete the integrated test of the above-mentioned potentiometer electric parameters.

\section{Working Principle of Integrated Test System}

The test system is an integrated system composed of potentiometer total resistance, zero resistance, dynamic noise and duplex potentiometer synchronization feature test. It is intended to be used in verification, inspection, selecting potentiometer for potentiometer manufacturers and machine manufacturers.

Definition of electric performance parameters of a potentiometer. A potentiometer has two fixed leading- out terminals (terminal $1 \& 3$ ) and one moving contact leading-out terminal (terminal 2). Potentiometer total resistance is defined as the resistance value between terminal 1 and 3 . When any potentiometer is demarcated, calibration of nominal resistance is required. At the same time, allowable deviation of the nominal resistance should be stipulated, and the total resistance value should be within the range of allowable deviation. Zero resistance is defined as the minimum resistance between terminal $2 \& 1$ and between $2 \& 3$ when the moving contact is near terminal $1 \& 3$. They are respectively called front and rear zero resistance. Dynamic noise refers to the electrical noise which does not exit in the input during the electrical signal output of potentiometer 1 and 2 when the contact point is moving on the resistive body, because of the irregular changes of contact resistance and because of the uneven resistance distribution on the resistive body, with mill volts as the measuring unit. Synchronization error of a duplex potentiometer is defined as the degree of difference between the output voltage and the input voltage ratio of the potentiometer's each resistive body at a specific position. According to IEC, synchronization error is expressed with the difference of an arbitrary potentiometer's attenuation decibels $\left(20 \lg \frac{V_{1-2}}{V_{1-3}}\right)$ and a benchmark league potentiometer's 
attenuation decibels ( $20 \lg \frac{V_{1^{\prime}-2^{\prime}}}{V_{1^{\prime}-3^{\prime}}}$ ). In normal use condition, input voltage of each potentiometer league is the same, i.e. $V_{1-3}=V_{1^{\prime}-3^{\prime}}$, thus synchronization error $\delta=20 \lg \frac{V_{1-2}}{V_{1^{\prime}-2^{\prime}}}$. Synchronization error is one of the key indicators to assess duplex potentiometer and the parameter has direct effect on the use of the whole electronic machine. ${ }^{[1]}$

Hardware composition of integrated test system and testing principles of electric parameters. In order to improve testing efficiency, multi-CPU parallel working mode is used in the integrated test system $^{[2] ~[3]}$. The composing structure of the system is shown in figure 1. Test module 1 is controlled by CPU1, which is in charge of the test of single and double potentiometer total resistance, zero resistance and synchronization error; test module 2 is controlled by CPU2, which is in charge of testing the dynamic noise of single and double potentiometer; master CPU is in charge of communicating with test module $1, \& 2$, which sends out test command, receives real-time test data, displays the test results dynamically and visually, and gives out sound and light indication after comparing the test results with the corresponding preset parameter values. Figure 2 is the test data and graphical display of a type-148 duplex potentiometer with nominal resistance of $50 \mathrm{~K}$. In the figure, the upper part is the tested potentiometer parameters' measured values, and the lower part is the "travel-synchronous" two-dimensional curve that reflects the synchronization error of the potentiometer within the given electrical route. From the figure, it can be seen that at the attenuation decibels $(48.7 \mathrm{~dB})$ point of the benchmark league potentiometer, the synchronization error is the largest, with $+2.1 \mathrm{~dB}$. Master CPU adopts microcontroller STC12C5A60S2 with double serial ports, so as to make real-time communication with testing

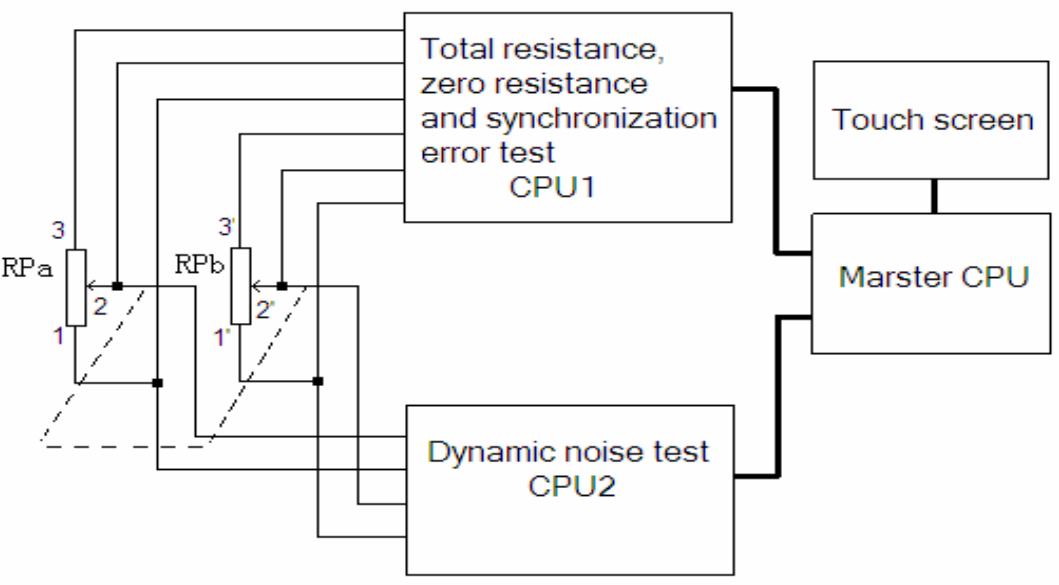

Figure1. Integrated test system composition structure diagram

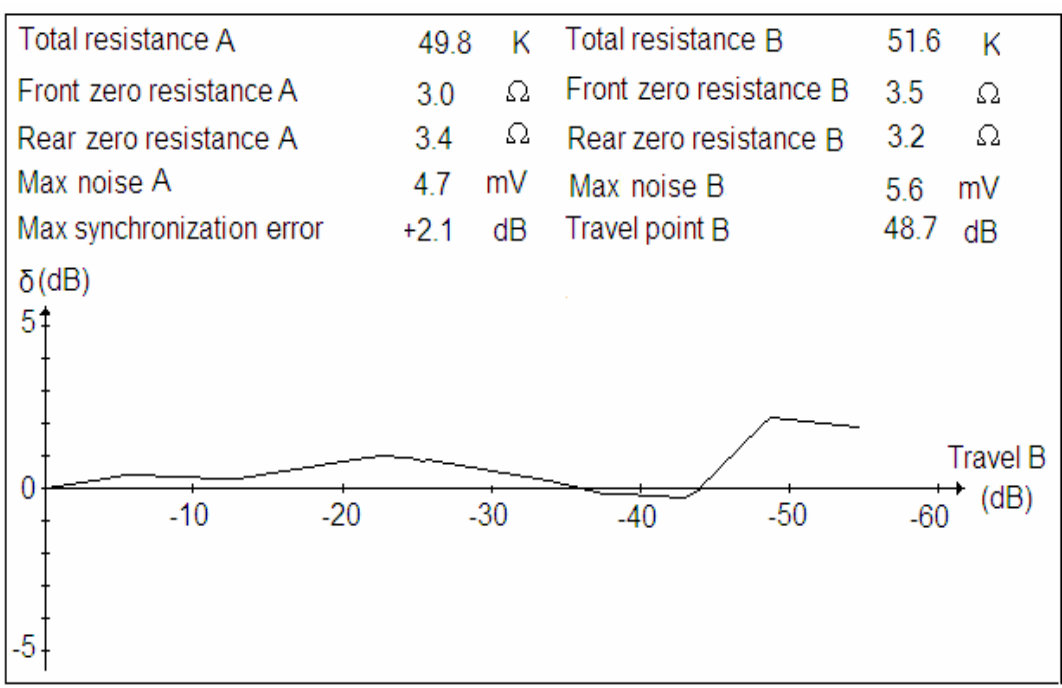

Figure2.Measured parameter values and graphical display interface module 1 and $2^{[4]}$.

The hardware structure of test module is shown in figure $3^{[5]}$. While total resistance of the potentiometer is being tested, electronic switch $K v$ is on, and $10 \mathrm{~V}$ voltage from constant voltage source circuit is added to terminal 1 and 3 of the tested potentiometers $R P a$ and $R P b$. And then sampling resistor $(R s)$ will produce sampling voltage $(\mathrm{V})$, and this voltage will pass the signal processing circuit and arrive at the input end of multi-channel switch. According to formula $R=\frac{10}{V} \times R_{s}$, total resistance of the measured potentiometer can be figured out. The range control signal outputted by CPU1 is used for the transition of electric switch, so as to choose sampling resistor (range resistor) 
of different resistance values, and thus the measurement accuracy will not be affected because of the small sample voltage. Of course, it should not exceed the analog input voltage limit of the A/D converter. When the movable contact moves within the range of the potentiometer's total electrical travel route, by continuously sampling the voltage at terminal $1 \& 2$ and 1'\& 2'of the coaxial double potentiometer, we can figured out (according to formula $\delta=20 \lg \frac{V_{1-2}}{V_{1^{\prime}-2^{\prime}}}$ ) the synchronization error of the duplex potentiometer corresponding to each travel point. With travel route as Axle $\mathrm{X}$ and synchronization error as Axle Y, we can draw a synchronization curve within the total travel range (as shown in figure 2). When the movable contact moves near terminal 1 or 3 on the resistor body, under the action of the voltage/current control signal outputted by CPU1, electronic switch $K v$ disconnected, $K i$ is connected, and the constant current source circuit provides electric current $5 \mathrm{~mA}$, thus front and rear zero resistance of the tested potentiometer can be figured out based on formula $R=\frac{V}{5 m A}$. In the formula, $V$ stands for the voltage produced by electric current when it passes through the zero resistance, and this voltage will pass the voltage follower and arrive at multi-channel switch input ${ }^{[6]}$. At different stages of the whole test process, A/D channel control signal outputted by CPU1 acts on the analog multiplexers, and corresponding input voltage is selected for A/D conversion. A/D converter adopts 24 -Bit $\Sigma$ - $\triangle \mathrm{AD} 7190$ with high speed, high precision and low noise ${ }^{[7]}$, so as to meet the requirements of the potentiometer electric parameter test for speed and accuracy. When

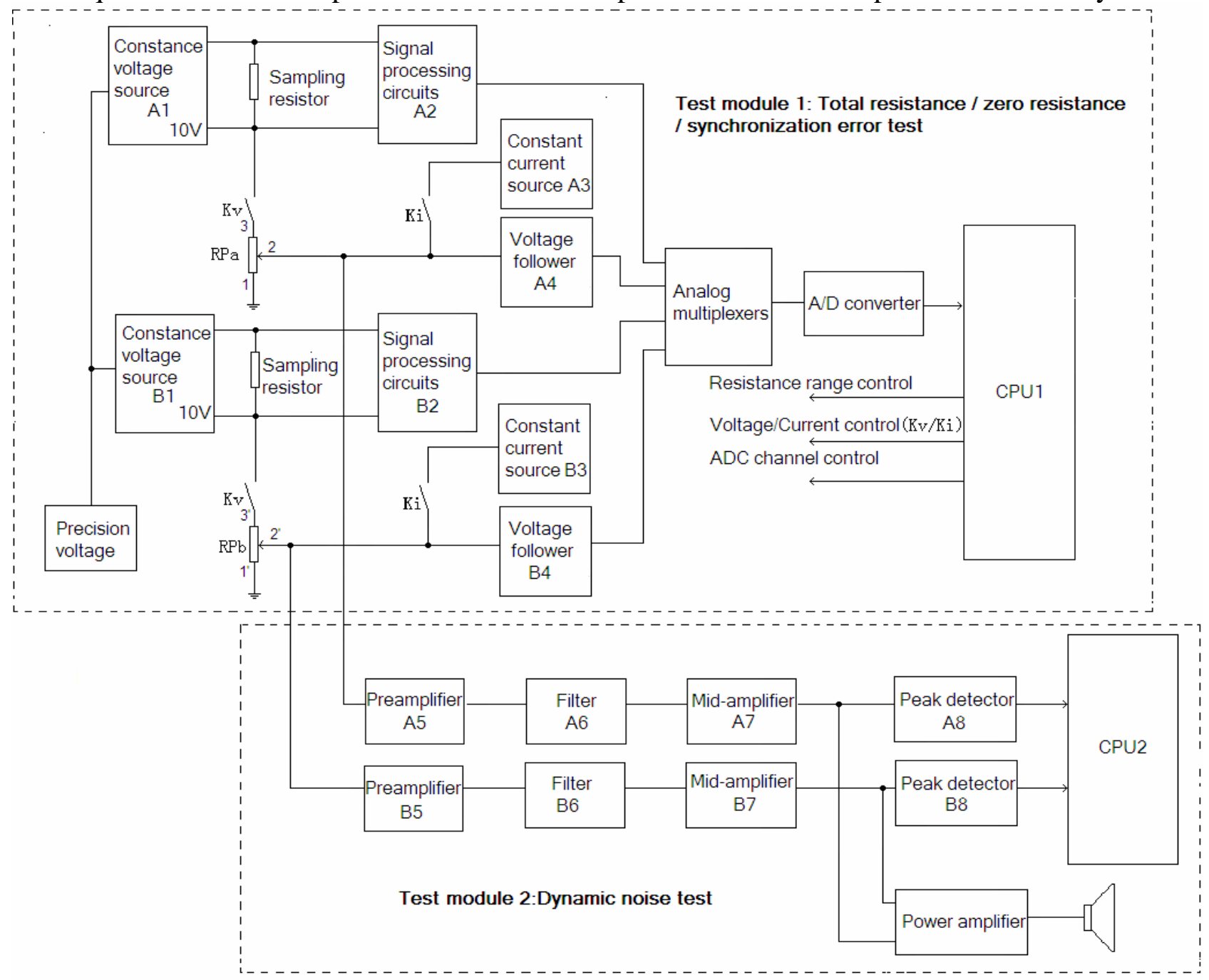

Figure3. Hardware block diagram of the test module 1, 2

test module 1 is in working state, the electric noise signals which are produced by the movable contact while moving on the potentiometer resistor body will be regulated and amplified by preamplifier, filter and mid-amplifying circuit, and then on the one hand they will enter CPU2's built-in A/D 
converter through peak detection and make analog/digital conversion. On the other hand, they can make loudspeaker produce sound through power amplifier circuit, thus achieving the function of monitoring dynamic noise ${ }^{[8]}$. CPU1 adopts microcontroller STC90C54RD, and CPU2 adopts microcontroller STC12C5410AD ${ }^{[9][10]}$.

\section{Software Design of Integrated Test System}

Software of potentiometer electrical parameter integrated test system includes master CPU program and testing module 1 and 2 controlling program. The master controlling program is in charge of the preset of the tested potentiometer parameters, receiving of test data, processing of the data, liquid crystal display and after-test sound and light indicating. As soon as testing module 1 receives the preset parameters value from master controlling CPU, it selects corresponding sampling resistor through the electronic switch and is ready for testing. When detected potentiometer has been inserted into the special fixture, it can start testing total resistance, synchronization error, front and rear zero resistance and send real-time data to master controlling CPU, the program flow is shown in figure 4. Complete test period of a potentiometer begins from the moment when the potentiometer inserts the special fixture to the time when front and rear zero resistance is tested at least once. After finishing a period, end mark (0x1f) should be sent to the master controlling CPU. When breaking of potentiometer from the special fixture is detected, empty fixture mark $(0 \times 2 \mathrm{f})$ should be sent to the master controlling CPU, which indicates the end of one potentiometer test. Testing module 2 and 1 synchronously and continuously sample data of dynamic noise voltage and send them real time to master controlling CPU.

\section{Conclusion}

Integrated test system of potentiometer electrical parameter is a system that allows various electrical parameters of potentiometer to be tested in parallel, thus improving labor productivity. The adoption of visualizing touch screen makes presetting parameters and testing results dynamically display with "Sync - stroke curve", which directly reflects the synchronization error of a, b in double potentiometer during their whole electrical route. Testing personnel can also adjust the potentiometer at the same time according to the curve till the synchronous error reaches within a reasonable range. They can also listen to the dynamic sound (just a rough estimate) or observe the data on LCD. The system is convenient to operate and no special training is needed so it has commendable prospects of promotion and application. 


\section{Acknowledgement}

This research was supported by the Basic and Applied Basic Research Project of Jiangsu University of Technology ( No. KYY13016 )

\section{References}

[1] H. Shaoqiang, The basic properties of the potentiometer, Selection accordance and Using principles Vol.6, (1996), p. 42-48 in Electronic Components and Materials.

[2] W. Furui, Monolithic Microcomputer Control System Design Encyclopedia, Beijing: Beijing University of Aeronautics and Astronautics Press, 1999.

[3] Y. Tianfeng, SCM Application System Design and Simulation Debugging, Beijing: Beijing University of Aeronautics and Astronautics Press, 2005.

[4] Information on http://www.stcmcu.com

[5] Z. Hongsheng, Electronic Circuit Design Technology Base, Chengdu: University of Electronic Science and Technology Press, 2012.

[6] Y. Jingping, G. Younan, An Integrated Detector of Duplex Potentiometer Electrical Parameters, China: 2013204836901.0[P]. 2014-01-08.

[7] Information on http://www.analog.com

[8] Y. Jingping, G. Younan, A Digital-display Dynamic Noise Detector of Potentiometer, China: 2014200513440.0[P]. 2014-05-15.

[9] C. Guiyou, Enhanced 8051 Microcontroller Practical Development Technology, Beijing: Beijing University of Aeronautics and Astronautics press, 2009.

[10] T. Wanyang, The design of electronic batching control system based on microcontroller Vol.19, (2013), p. 18-19 in Electronics World. 